\title{
Expression of Hormone Receptor Status and HER-2/neu in Breast Cancers: A Review in Various Ethnic Groups
}

Nirmal Daniel ${ }^{1}$, Mangala Goneppanavar ${ }^{2}$, Sowmya Srinivasan ${ }^{3}$

\begin{abstract}
The role of hormone receptor status including estrogen receptor (ER), progesterone receptor (PR), and human epidermal growth factor receptor type 2 (HER-2/neu) commonly termed as ER, PR, and HER-2/neu expression in breast cancer by immunohistochemistry (IHC) is a widely accepted tool to assess prognosis as well as therapeutic management. With further standardization of the reporting template prescribed by the College of American Pathologists as "CAP Protocol" and American Society of Clinical Oncology ("ASCO guidelines"), IHC has effectively replaced cytological assays in evaluating the status of expression of hormonal receptors. The pattern of these hormonal receptors' expressions varies with regard to genetic, environment, lifestyle, and sociodemographic factors. There are well-established clinical evidences to substantiate the clinical utility of ER expression as a standard predictive biomarker to assess the prognosis of hormonal therapy. The same insight about the clinical utility of $\mathrm{PR}$ is questionable. However, the diagnostic utility of PR for predicting the clinical response to chemotherapy among ER-positive breast cancer patients remains unclear. This warrants future studies incorporating the integrated analysis of survival data, gene expression and its data profile, and compilation of ER and PR expressions from various large cohort analysis of breast cancer patients. This review focuses on the clinical utility and the inherent variation of the hormone receptor expression among notable diverse demographic study groups across the world.

Keywords: Breast cancer, HER-2/neu, Hormone receptors, Immunohistochemistry.

Annals of SBV (2020): 10.5005/jp-journals-10085-8116
\end{abstract}

\section{INTRODUCTION}

Cancer being the second leading cause of death worldwide has an incidence of approximately $70 \%$ deaths occurring in developing countries. ${ }^{1}$ In the past few decades, a substantial increase is noted in the incidence rate and cancer-associated sequelae in the Indian subcontinent. ${ }^{1,2}$ Among Indian females, breast cancer has ranked number among variable age groups and mortality rate ranging as 25.8 for every 100,000 women. ${ }^{3}$ The role of the histopathologist as a diagnostic oncologist is entitled to determine the biological behavior of tumor in terms of histological type, extent of differentiation, mitotic activity, microscopic lymphovascular invasion, and metastasis which remains the mainstay in diagnosis. ${ }^{4}$

The ASCO and CAP recommend the evaluation of estrogen, progesterone, and HER-2/neu markers by means of IHC for all newly diagnosed cases of breast cancer and recurrent cases. ${ }^{5}$ This prognostic and therapeutic information provided by conventional histopathology, and IHC represents the firm foundation upon which the treatment strategy is built.

The utility of these biomarkers in the scope of tumors of the breast has been studied by researchers worldwide. The purpose of this review article is to study the pattern of hormone receptors such as estrogen, progesterone, and HER-2/neu expressions among patients who underwent mastectomy. A literature search is done using the keywords breast cancer, expression of hormonal receptors, and HER-2/neu in databases such as PubMed and Scopus which revealed that 28 articles out of which 11 relevant full-text articles published in the time period between 1990 and 2018 are reviewed.

\footnotetext{
${ }^{1-3}$ Department of Pathology, Mahatma Gandhi Medical College and Research Institute, Sri Balaji Vidyapeeth, Puducherry, India

Corresponding Author: Sowmya Srinivasan, Department of Pathology, Mahatma Gandhi Medical College and Research Institute, Sri Balaji Vidyapeeth, Puducherry, India, Phone:+91 8903602824, e-mail: drssowmya@hotmail.com

How to cite this article: Daniel N, Goneppanavar M, Srinivasan S.
} Expression of Hormone Receptor Status and HER-2/neu in Breast Cancers: A Review in Various Ethnic Groups. Ann SBV 2020;9(1): 12-16.

Source of support: Nil

Conflict of interest: None

\section{Estrogen and Progesterone Receptors from a Historical Perspective}

Elwood Vernon Jensen isolated ERs and discovered their importance in breast cancers in the year 1958. He received the "Albert Lasker award" in 2004 for basic medical research for the discovery of hormone receptors. ${ }^{6}$ During the early 1980 s, Jensen published an original article which emphasized the fact that the identification of ER in the laboratory provided a platform for targeted site specificity of estrogenic effect in breast cancer. With more emphasis, a test was formulated to predict the clinical outcome of antihormonal therapy among the patients with breast cancer and a target was categorized to develop new drugs for the management. ${ }^{6}$ The ER in conjunction with PR was the first biological markers to be used in the management of breast cancer.

(0) The Author(s). 2020 Open Access This article is distributed under the terms of the Creative Commons Attribution 4.0 International License (https://creativecommons. org/licenses/by-nc/4.0/), which permits unrestricted use, distribution, and non-commercial reproduction in any medium, provided you give appropriate credit to the original author(s) and the source, provide a link to the Creative Commons license, and indicate if changes were made. The Creative Commons Public Domain Dedication waiver (http://creativecommons.org/publicdomain/zero/1.0/) applies to the data made available in this article, unless otherwise stated. 


\section{Methods of Estimation of Hormone Receptor Status \\ Cytosol Assays}

In the early 1970s, the mode of selection for determining the estrogen, progesterone, and HER-2/neu status was dextrancoated charcoal ligand binding assay commonly coined as DCC. The necessity for the use of radioactive components and the need for extensive quantity of freshly frozen tissue made it technically difficult to perform cytosol assays which was also time-consuming. ${ }^{7}$ An alternative was a simpler and cheaper enzyme immunoassay (EIA) which gradually replaced DCC with the development of the newer regimes of antibodies.

\section{Immunohistochemistry}

With technological advancements and improvised procedures for antigen retrieval and further exploration of the selection of anti-ER and anti-PR antibodies, the immunohistochemical assay became an essential alternative. The ability of IHC assays to be carried out on routine histopathological microscopic sections even in the smallest and sparsely cellular tumors was an added advantage. ${ }^{7}$ The major disadvantages of the IHC are the subjective method of analyzing the staining interpretation and in determining the benchmark range value to categorize between receptor-positive and receptornegative tumors. Reassuringly, Zafrani et al. in a cross-sectional analysis of 793 cases showed that IHC is more accurate and efficient in terms of sensitivity and specificity than the cytosolic assays. ${ }^{8}$

\section{Reporting the Results of Biomarker TESTING}

In 2010, the ASCO and CAP proposed Guideline Recommendations for Estrogen, Progesterone, and HER-2/neu IHC Testing in breast cancer. In this guideline, it was summed up that up to $20 \%$ of IHC determinations of ER and PR testing performed worldwide could be incorrect, with high incidence for false-negative and false-positive results. ${ }^{5}$ There has been particular concern that among breast cancer patients; faintly, ER-positive tumors may deliberately be categorized to be ER-negative, thus resulting in these groups being obviated for the potential beneficiary from antiestrogen therapy or vice versa. The major concerns with testing owed to the variation in pre-analytic variables, thresholds for positivity range, and data interpretation specifications and criteria. Recent literature, however, suggests that interobserver variations in calculating scores of ER as weak, intermediate, and strong positivity is at times misguiding and has differing therapeutic implications. ${ }^{9}$ This led to the formulation of IHC scoring system and biomarker reporting template to assist pathologists in the useful reporting of relevant information taking into account clinician prerogative, institutional standard operating procedures, and individual preferences.

\section{Allred System of Scoring for ER and PR}

The Allred scoring system was introduced in order to reduce the number of borderline cases and to categorize them into either one of the positive or negative groups. It is a semiquantitative system of scoring that calculates the proportion of positive cells (scored on a scale of $0-5$ ) and staining intensity (scored on a scale of $0-3) .{ }^{10}$ The proportion of expression and intensity grade will be then summed to arrive a total score ranging from 0 to 2 through 8. A score ranging from 0 to 2 was regarded as negative while 3 to 8 as positive (Table 1$)^{9}$
Table 1: Guidelines for interpretation of ER and PR results by Allred method

\begin{tabular}{llll}
\hline $\begin{array}{l}\text { Proportion } \\
\text { score (PS) }\end{array}$ & Observation & $\begin{array}{l}\text { Intensity score } \\
(\text { IS) }\end{array}$ & Observation \\
\hline 0 & None & 0 & None \\
1 & $1 \%$ & 1 & Weak \\
2 & $1-10 \%$ & 2 & Intermediate \\
3 & $10-33 \%$ & 3 & Strong \\
4 & $33-66 \%$ & - & - \\
5 & $66-100 \%$ & - & - \\
Total score (sum of PS and IS) & & Interpretation \\
$\quad 0-2$ & & & Negative \\
$3-8$ & & & Positive \\
\hline
\end{tabular}

\section{HER-2/NEU TESTING}

The human epidermal growth factor (HER) group of receptors takes part in cellular proliferation and differentiation via multiple signaling cell pathways. The family comprises four receptors, namely HER-1, HER-2, HER-3, and HER-4 (also called ErbB1, ErbB2, ErbB3, and ErbB4, respectively). ${ }^{11}$ The ErbB is coined from the term erythroblastic oncogene $B$, a gene isolated from avian genome. This HER2 oncogene (in humans) is inbuilt with a transmembrane glycoprotein coded with tyrosine kinase activity known as p185 which was discovered by a committee of Research Scholars at Harvard University in $1984 .^{11}$

Around $15 \%$ of invasive breast cancer lesions exhibit HER2 gene amplification and overexpression coded proteins. ${ }^{11}$ HER-2/ neu is used as the predictive marker for targeted therapies such as humanized monoclonal antibody drug regime like trastuzumab which has resulted in significant improvement in overall prognosis and disease-free survival, as well as decreased rate in relapse and metastasis, among patients with early HER-2/neu-positive tumors. ${ }^{12}$ Several methods can detect HER-2/neu overexpression or amplification which includes immunohistochemical methods, blotting techniques to analyze protein expression, in situ hybridization (ISH) procedures, polymerase chain reactions, etc. ${ }^{13}$

\section{IHC Scoring System}

The ASCO/CAP has recently published guidelines with upgraded recommendations for HER-2/neu analysis in breast cancer patients. According to the revised 2018 recommendation from ASCO/CAP joint committee in 2018, HER-2/neu showing a grade of (3+) is defined as cells showing more than $10 \%$ of invasive tumor cells with complete intense membrane staining and is positive. HER-2/ neu IHC (2+) is weak to moderate to complete membranous, a score of $2+$ is assumed to be equivocal, whereas negative for HER-2/neu expression includes no staining expression or staining that is barely perceptible in less than $10 \%$ of the invasive tumor cells ${ }^{5}$ However, an equivocal result (score $2+$ ) warrants further exploration as reflex test (same specimen using ISH) or to place an order for a newer test (new specimen if available using IHC or ISH). ${ }^{13}$

Many laboratory directors have concluded that the best approach to HER-2/neu testing from the point of view of costeffectiveness is to start with the immunohistochemical procedure, which is scored according to the recent ASCO/CAP guideline. If the results are either $3+$ or $0 / 1+$, the determination can safely stop there, since the correlation with gene amplification or lack of it, respectively, as measured by $\mathrm{FISH}$, is nearly $100 \%$. If the IHC is $2+$ 
(equivocal), the performance of FISH is recommended, and the result obtained tends to be regarded as the definitive result. Other laboratories perform both IHC and FISH on all the cases because of the small possibility of a patient being HER-2/neu positive by one or other of the test.

\section{ISH Scoring System}

Interestingly, HER-2/neu analysis which evaluates protein expression via antibody binding and HER-2/neu ISH determines the number of parent gene copy with the help of a DNA probe. Several diagnostic systems had been implemented in an attempt to couple with the DNA probe, including fluorescent, chromogenic, or silver-based systems. ${ }^{14}$ In general, HER2:CEP17 ratio had been assumed to be better in reflecting the true HER2 amplification status than HER2 copy as standalone. ${ }^{14}$ The ASCO and CAP have proposed the guidelines for documenting the observations of HER-2/neu testing by ISH employing single-probe as well as dual-probe assays. ${ }^{15}$

\section{Molecular Classification of Breast Cancers}

Depending upon the hormone receptor status and HER-2/neu, breast cancers are categorized into four different domains.

\section{Luminal Type A}

These types of cancers, as the name describes, are positive for hormone receptors (ER and PR) and negative for HER-2/neu and/ or low Ki67 index. About $80 \%$ of breast cancers are ER expression positive, and about $65 \%$ of these are also PR expression positive which means that they grow in response to estrogen and progesterone, respectively. These tumors are more responsive to hormone therapy than hormone receptor-negative tumors. ${ }^{16}$

\section{Luminal Type B}

This term is used to describe the cancers that are ER+, PR+, HER-2/ neu+, and/or high Ki-67 index. These cancers can be treated with hormone therapy as well as drugs that target HER-2/neu. ${ }^{16}$

\section{HER2 Enriched}

About $20 \%$ of breast cancers are HER-2/neu+. These cancers are very aggressive and fast growing with a high tumor grade. They do not respond to hormone therapies; instead, they are treated with antibodies against HER-2/neu receptors (Herceptin) blocking their action. Females with the expression of HER-2/ neu-positivity-type tumors are usually noted at a younger age than the other two luminal domains. HER-2/neu amplification coincides with inverse proportion with that of estrogen and progesterone expressions. ${ }^{16}$

\section{Basal-like or Triple-negative Phenotype}

Almost $10-20 \%$ of breast cancers are triple-negative type based on the nonexpression of markers, commonly referred to as basal-like tumor. Most breast cancers associated with BRCA-1 gene fall under this category usually containing p53 gene mutations. Although these cancers respond fairly well to chemotherapy, they tend to recur. No perfect therapy has been devised so far, but several promising strategies are being aimed at triple-negative breast cancers. However, it should be noted that most triple-negative breast tumors are not basal-like and vice versa. ${ }^{16,17}$

\section{Clinical Relevance of the Hormone RECEPTORS}

The ER being the most efficient biomarker in breast cancer ranges with an incidence of $75 \%$ among overall breast cancers comprising the majority of the luminal-type tumors. The tumor under this category usually is of lower grade with relatively good prognosis. ${ }^{14}$

The development of tamoxifen for the treatment of ER-positive breast cancers has resulted in improved survival in the patients. ${ }^{6}$ Following the beneficial action of tamoxifen in advanced disease, several randomized trials had been worked upon to assess its efficacy as an adjuvant systemic treatment for primary disease. The results of the majority of the trials carried out worldwide have been portrayed in several overview analyzes. ${ }^{6,18}$ ER and PR have become reference points for the other markers so that more accurate information as to receptor status will enhance the quality of research into the biology of breast cancer and its diversity.

The tumors expressing both of these receptors show good prognosis with favorable response to treatment especially with hormones. ${ }^{19}$

\section{Studies Correlating ER, PR, and HeR-2/ neu Status in Breast Cancers}

Observation done by Desai et al. on immunohistochemical hormone receptor status of breast cancer in the Indian subcontinent comprises 798 tumors. Out of the 798 breast tumors studied, $30 \%$ of tumors were ER-positive and $46.1 \%$ were PR-positive. A significant proportion of the tumors (46.5\%) were noted to be receptor-negative. ${ }^{20}$ A similar Indian study by Redkar along with his researchers also noted a higher incidence of hormone receptor non-reactivity among females with breast cancer. ${ }^{21}$ Such results are partially explained by the fact that the study group comprises younger premenopausal women with median age of 48 years presenting with tumors of higher grade. Young patients possess higher levels of circulating estrogens in their body and a corresponding low expression of steroid receptor which is reflected in their tumors.

Another study by Mudduwa among 151 breast cancer cases in Sri Lanka demonstrated $45.7 \%$ ER and a $48.3 \%$ PR reactivity. ${ }^{18}$ The major crux of the patients included in the study showed highgrade tumors, thereby explaining the low prevalence of hormone receptor expression.

Another researcher Kaul with his coworkers in a study conducted on 55 cases of breast cancer in the parts of Himachal Pradesh with the aim of evaluating hormone receptor status and to compare with previously published data from other centers in India, which showed ER and PR positivity of $34.5 \%$ and $36.4 \%$, respectively. ${ }^{22}$

Chariyalertsak et al. reported the results concurring to observations of Desai et al. with 36.1\% ER and 45.8\% PR reactivity among 83 breast cancer cases in Thailand. ${ }^{23}$ In contradictory, a cross-sectional study conducted in China showed $73.5 \%$ and $65.5 \%$ of ER and PR reactivity in their analysis of 200 breast cancers. ${ }^{24}$ Hormone receptor positivity in breast cancers among Asian population has been found to be lower than the Western world. Christopher et al. have documented a prevalence of $76-78 \%$ of hormone receptor-positive breast cancers in the USA from 1992 to 1998 with a rise in the prevalence over the years. ${ }^{25}$ Barnes et al. among a population of 170 breast cancer patients in London in 
the early 1990s showed $65 \%$ of cases to be ER-positive using immunohistochemical assays. ${ }^{7}$ This can be attributed partially to the dietary, lifestyle, and genetic factors among the two ethnic groups which further needs corroborative evidence supported by large cohort studies.

A study by Hefti et al. which employed gene expression profiling procedure along with the clinical and immunohistochemical data across two large and diverse datasets clearly mentioned that ER-negative and PR-positive breast cancers are not a reproducible subtype. It also mentions that PR expression is not associated with prognosis in ER-negative breast cancer. ${ }^{26}$ It is essential to document that testing for PR expression currently provides no clinically actionable information in ER-positive breast cancer, as patients will receive endocrine therapy regardless of PR status. There is no consensus as to whether knowledge of PR expression by IHC has a role in informing the use of chemotherapy in ER-positive breast cancer.

With regard to HER-2/neu status, Dawood et al. in an institutional-based review in the University of Texas among 2091 women with breast cancer diagnosed from 1991 to 2007 studied whether trastuzumab improves prognosis in 15\% cases demonstrating HER-2/neu positivity. ${ }^{27}$

A 5-year retrospective study conducted by Siddiqui et al. in Northern India has noted a remarkably higher $62 \% \mathrm{HER}-2 /$ neu positivity. The author also claims the result to be attributed to predominantly younger patient population in their study group. ${ }^{28}$

\section{Conclusion}

From the review, it is apparent that the presentation and behavior of breast cancer in India may be varied due to differences in inherent, environment, lifestyle, sociodemographic structure, and ethnic race. The reason for increased incidence rates of breast cancer in premenopausal women is not very well understood but a major factor could be underdiagnosis and under-reporting among the elderly population.

The CAP and ASCO recommend ER and PR testing in all newly diagnosed cases of breast cancer. However, it is important to note that testing for PR expression currently provides no clinically significant information as patients will receive endocrine therapy for all ER-positive tumors regardless of the PR status. In developing countries with resource-poor laboratory setting and increased health care needs, removing PR testing from the routine diagnostic workup of invasive breast cancer could save the health care industry substantial funds with no loss in the clinical utility of the pathological evaluation.

\section{References}

1. Ferlay J, Soerjomataram I, Dikshit R, Eser S, Mathers C, Rebelo M, et al. Cancer incidence and mortality worldwide: sources, methods and major patterns in GLOBOCAN 2012. Int J Cancer 2015;136(5):E359E386. DOI: 10.1002/ijc.29210.

2. Malvia S, Bagadi SA, Dubey US, Saxena S. Epidemiology of breast cancer in Indian women. Asia Pac J Clin Oncol 2017;13(4):289-295. DOI: 10.1111/ajco.12661.

3. Gupta A, Shridhar K, Dhillon PK. A review of breast cancer awareness among women in India: cancer literate or awareness deficit? Eur J Cancer Oxf Engl 1990 2015;51(14):2058-2066. DOI: 10.1016/j. ejca.2015.07.008.

4. Leong AS-Y, Zhuang Z. The changing role of pathology in breast cancer diagnosis and treatment. Pathobiology 2011;78(2):99-114. DOI: 10.1159/000292644.
5. Hammond MEH, Hayes DF, Dowsett M, Allred DC, Hagerty KL, Badve $S$, et al. American Society of Clinical Oncology/college of American Pathologists Guideline recommendations for immunohistochemical testing of estrogen and progesterone receptors in breast cancer (unabridged version). Arch Pathol Lab Med 2010 Jul;134(7):e48-e72. DOI: $10.1200 / J C O .2009 .25 .6529$.

6. Jensen EV, Jordan VC. The estrogen receptor: a model for molecular medicine. Clin Cancer Res Off J Am Assoc Cancer Res 2003;9(6): 1980-1989.

7. Barnes DM, Hanby AM. Oestrogen and progesterone receptors in breast cancer: past, present and future. Histopathology 2001;38(3):271-274. DOI: 10.1046/j.1365-2559.2001.01060.x.

8. Zafrani B, Aubriot MH, Mouret E, de Cremoux P, De Rycke Y, Nicolas $A$, et al. High sensitivity and specificity of immunohistochemistry for the detection of hormone receptors in breast carcinoma: comparison with biochemical determination in a prospective study of 793 cases. Histopathology 2000;37(6):536-545. DOI: 10.1046/j.13652559.2000.01006.x.

9. Colleoni M. Chemotherapy is more effective in patients with breast cancer not expressing steroid hormone receptors: a study of preoperative treatment. Clin Cancer Res 2004;10(19):6622-6628. DOI: 10.1158/1078-0432.CCR-04-0380.

10. Qureshi A, Pervez S. Allred scoring for ER reporting and it's impact in clearly distinguishing ER negative from ER positive breast cancers. JPMA J Pak Med Assoc 2010;60(5):350-353.

11. Iqbal N, Iqbal N. Human epidermal growth factor receptor 2 (HER2) in cancers: overexpression and therapeutic implications. Mol Biol Int 2014;2014:1-9. DOI: 10.1155/2014/852748.

12. Verma S, Lavasani S, Mackey J, Pritchard K, Clemons M, Dent S, et al. Optimizing the management of her2-positive early breast cancer: the clinical reality. Curr Oncol 2010;17(4):20-33. DOI: 10.3747/co.v17i4.700.

13. Wolff AC, Hammond MEH, Allison KH, Harvey BE, Mangu PB, Bartlett $J M S$, et al. Human epidermal growth factor receptor 2 testing in breast cancer: American Society of Clinical Oncology/College of American Pathologists Clinical Practice Guideline focused update. Arch Pathol Lab Med 2018;142(11):1364-1382. DOI: 10.5858/arpa.2018-0902-SA.

14. Han G. ER, PR and HER2 testing in breast cancer. Diagn Histopathol 2014;20(11):440-445. DOI: 10.1016/j.mpdhp.2014.10.002.

15. Hammond MEH, Hayes DF, Dowsett M, Allred DC, Hagerty KL, Badve $\mathrm{S}$, et al. American society of clinical oncology/college of american Pathologists guideline recommendations for immunohistochemical testing of estrogen and progesterone receptors in breast cancer. J Clin Oncol 2010;28(16):2784-2795. DOI: 10.1200/JCO.2009.25.6529.

16. Tsang JYS, Tse GM. Molecular classification of breast cancer. Adv Anat Pathol 2019. 1.

17. Russnes HG, Lingjærde OC, Børresen-Dale A-L, Caldas C. Breast cancer molecular stratification: from intrinsic subtypes to integrative clusters. Am J Pathol 2017;187(10):2152-2162. DOI: 10.1016/j. ajpath.2017.04.022.

18. Mudduwa LKB. Quick score of hormone receptor status of breast carcinoma: Correlation with the other clinicopathological prognostic parameters. Indian J Pathol Microbiol 2009;52(2):159-163. DOI: 10.4103/0377-4929.48906.

19. Viale G, Regan MM, Maiorano E, Mastropasqua MG, Dell'Orto P, Rasmussen BB, et al. Prognostic and predictive value of centrally reviewed expression of estrogen and progesterone receptors in a randomized trial comparing letrozole and tamoxifen adjuvant therapy for postmenopausal early breast cancer: BIG 1-98. J Clin Oncol Off J Am Soc Clin Oncol 2007;25(25):3846-3852. DOI: 10.1200/ JCO.2007.11.9453.

20. Desai SB, Moonim MT, Gill AK, Punia RS, Naresh KN, Chinoy RF. Hormone receptor status of breast cancer in India: a study of 798 tumours. The Breast 2000;9(5):267-270. DOI: 10.1054/brst.2000.0134.

21. Redkar AA, Kabre SS, Mittra I. Estrogen \& progesterone receptors measurement in breast cancer with enzyme-immunoassay \& correlation with other prognostic factors. Indian J Med Res 1992;96: $1-8$. 
22. Kaul R, Sharma J, Minhas SS, Mardi K. Hormone receptor status of breast cancer in the himalayan region of Northern India. Indian J Surg 2011;73(1):9-12. DOI: 10.1007/s12262-010-0121-5.

23. Chariyalertsak S, Chariyalertsak S, Ruangvejvorachi P. Immunohistochemical detection of estrogen and progesterone receptors in primary breast cancer. Asian Pac J Allergy Immunol 1998;16(4):161-166.

24. Lu X, Chen S, Huang S. A study on methodology and the criteria for positive immunohistostaining of estrogen and progesterone receptors in paraffin embedded sections of breast cancer. Zhonghua Bing Li Xue Za Zhi 1996;25(6):329-331.

25. Li Cl, Daling JR, Malone KE. Incidence of invasive breast cancer by hormone receptor status from 1992 to 1998. J Clin Oncol Off J Am Soc Clin Oncol 2003;21(1):28-34. DOI: 10.1200/JCO.2003.03.088.
26. Hefti MM, Hu R, Knoblauch NW, Collins LC, Haibe-Kains B, Tamim $\mathrm{RM}$, et al. Estrogen receptor negative/progesterone receptor positive breast cancer is not a reproducible subtype. Breast Cancer Res [Internet] 2013;15(4). DOI: 10.1186/bcr3462Available from: http://breast-cancer-research.biomedcentral.com/articles/10.1186/ bcr3462.

27. Dawood S, Broglio K, Buzdar AU, Hortobagyi GN, Giordano SH. Prognosis of women with metastatic breast cancer by HER2 status and trastuzumab treatment: an institutional-based review. J Clin Oncol 2010;28(1):92-98. DOI: 10.1200/JCO.2008.19.9844.

28. Siddiqui B, Piyush AR, Faridi SH, Ahmad SS, Khan R. Trends in molecular classification of breast carcinoma in a tertiary health care centre: a 5 year retrospective study. Int Arch Biomed Clin Res 2016;2(2):69-72. DOI: 10.21276/iabcr.2016.2.2.15. 